\title{
SUL FUNZIONAMENTO DEL ROCCHETTO DI INDUZIONE CON GLI INTERRUTTORI DI TIPO RECENTE.
}

\author{
Nota di O. M. CORBINO.
}

1. - L'uso dei moderni tipi di interruttori rotativi rapidi nei quali la corrente primaria viene stabilita e interrotta da un getto rotante di mercurio in seno a un gas inerte e con grande frequenza, ha profondamente modificato le condizioni antiche di funzionamento del rocchetto d'induzione. Auzitutto la rapidità della rottura permette di fare a meno del condensatore, cosicchè la teoria della fase di apertura si semplifica riducendosi questa a una scarica senza oscillazioni. In secondo luogo la frequeñza con la quale si susseguono le chiusure e le aperture fa sì che la corrente primaria viene interrotta prima che abbia raggiunto il valore di regime, e ristabilita. spesso mentre ancora sussiste la corrente secondaria destat: dalla precedente rottura.

Ottenere dal rocchetto, in tali condizioni, correnti secondarie intense e assolutamente unilaterali, come occorrono, ad esempio, nella radiografia intensiva, richiede un esame accurato teorico e sperimentale del funzionamento del rocchetto. Questo esame è stato iniziato e approfondito da qualche tempo con varie ricerche mie e del dott. Trabacchi; esse ei hanno condotto alla costruzione di alcuni apparecchi per raggi $X$ il cui successo tecnico è essenzialmente collegato coi risultati di quegli studi che noi andremo man mano esponendo e che serviranno di illustrazione alle memorie puramente descrittive. già pubblicate.

In questa nota sarà dedotta la legge di svolgimento della corrente secondaria e la potenza ottenibile al secondario, nell 
ipotesi che il ricevitore abbia, come $\mathrm{i}$ tubi per raggi $\mathrm{X}$ non mollissimi, una caratteristica di tipo lineare $(\mathrm{V}=a+b i$, dove $\mathrm{V}$ è la tensione, $i$ la corrente, $a$ e $b$ due costanti); e che per virtù dell' interruttore e del sistema adottato di due avvolgimenti primari atti a produrre magnetizzazioni opposte del nucleo, si succedano rapidamente chiusure e rotture del circuito primario con funzionamento alternativo dei due avvolgimenti. Sarà così dimostrato che l'artificio di far seguire a una rottura una chissura atta a magnetizzare il nucleo in senso opposto, artificio che noi abbiamo usato nel nostro apparecchio, non può condurre da solo a buoni risultati, senza le precauzioni di cui la teoria e l'esperienza hanno rivelato la necessità e l'importanza.

Per non complicare l'esposizione ammetterò che siano trascurabili le fughe magnetiche fra primario e secondario, ciò che non altera le conclusioni qualitative sull'andamento dei fenomeni.

Con ciò le equazioni che dominano lo svolgimento delle correnti secondarie nella fase di chiusura sono assai più semplici di quelle risolute nella mia prima memoria del 1908 ').

Chiamando L la self del primario, $m$ il coefficiente di moltiplicazione del rocchetto, eguale al rapporto fra i numeri delle spire secondarie e primarie, $M$ il coefficiente di induzione mutua; E la f.e. $m$. costante agente nel primario, $r_{1}$ ed $r_{2}$ le resistenze ohmiche del primario e del secondario, si avrà

$$
\begin{aligned}
& r_{1} i_{1}+\mathrm{L} \frac{d i_{1}}{d t}+\mathrm{M} \frac{d i_{2}}{d t}=\mathrm{E} \\
& r_{2} i_{2}+m^{2} \mathrm{~L} \frac{d i_{3}}{d t}+\mathrm{M} \frac{d i_{1}}{d t}+a+b i_{2}=0
\end{aligned}
$$

poichè la self del secondario è $m^{2}$ volte quella del primario. L' ultima equazione, ponendo

$$
r_{2}+b=r
$$

i) O. M. Corbino; Nuovo Cimento, vol. 15, p. 303, 1908. 
può essere scritta

$$
r i_{2}+m^{2} \mathrm{~L} \frac{d i_{2}}{d t}+\mathrm{M} \frac{d i_{1}}{d t}=-a
$$

e inoltre, osservando che $\mathrm{M}=m \mathrm{~L}$, dalle due equazioni relative al primario e al secondario si possono ottenere queste altre nelle quali le correnti $i_{1}$ e $i_{2}$ sono separate in due equazioni distinte :

Posto

$$
\begin{aligned}
& r_{1} i_{1}+\mathrm{L} \frac{r+m^{2} r_{1}}{r} \frac{d i_{1}}{d t}=\mathrm{E} \\
& r i_{2}+\mathrm{L} \frac{r+m^{2} r_{1}}{r_{1}} \frac{d i_{2}}{d t}=-a .
\end{aligned}
$$

$$
\alpha=\frac{r_{1} r}{\mathrm{~L}\left(r+m^{2} r_{1}\right)}
$$

queste equazioni hanno per soluzione

$$
\begin{aligned}
& i_{1}=\mathrm{A}+\mathrm{B} e^{-x l} \\
& i_{2}=\mathrm{C}+\mathrm{D} e^{-\alpha l}
\end{aligned}
$$

dove $\mathrm{A}, \mathrm{B}, \mathrm{C}, \mathrm{D}$ sono costanti da determinare con le condizioni ai limiti.

Cominciando da $i_{1}$ si osservi che $i$ valori di $A$ e $B$ sono indipendenti dal fatto che la chinsura sussista per un tempo breve o lunghissimo. Ma in quest' ultimo caso si avrebbe, per $t$ grandissimo,

$$
i_{1}=\frac{\mathrm{E}}{r_{1}}, \quad \text { ciò̀ } \mathrm{A}=\frac{\mathrm{E}}{r_{1}}
$$

Inoltre la quantità di elettricita passata in meno per il processo induttivo dopo un tempo lunghissimo, deve essere eguale alla variazione del flusso divisa per $r_{1}$. Se all'istante della chiusura sopravvive nel secondario la corrente di apertura anteriore, col valore $I_{\tau}$, e se la nuova chiusura tende a produrre una magnetizzazione di senso opposto, il flusso nel primario varierà da $\mathrm{MI}_{\tau} \mathrm{a}-\mathrm{L} \frac{\mathrm{E}}{r_{1}}$; 
e perciò

$$
\int_{0}^{\infty} \mathrm{B} e^{-x t} d t=-\frac{\mathrm{B}}{\alpha}=\frac{\mathrm{MI_{ \tau } + \mathrm { L }} \frac{\mathrm{E}}{r_{1}}}{r_{1}}=\frac{\mathrm{L}}{r_{1}}\left(m \mathrm{I}_{\tau}+\frac{\mathrm{E}}{r_{1}}\right)
$$

da cui

$$
\mathrm{B}=-r \frac{m \mathrm{I}_{\tau}+\frac{\mathrm{E}}{r_{1}}}{r+m^{2} r_{1}}
$$

Sarà perolò

$$
i_{1}=\frac{\mathrm{E}}{r_{1}}-\frac{r}{r+m^{2} r_{1}}\left(m \mathrm{I}_{\tau}+\frac{\mathrm{E}}{r_{1}}\right) e^{-\alpha t} .
$$

Se invece la chiusura determinasse un campo magnetico. nel medesimo senso del precedente, come nei dispositivi, anteriori sarebbe

$$
\int \mathrm{B} e^{-x t} d t=\frac{\mathrm{MI}_{\tau}-\mathrm{L} \frac{\mathrm{E}}{r_{1}}}{r_{1}}=\frac{\mathrm{L}}{r_{1}}\left(m \mathrm{I}_{\tau}-\frac{\mathrm{E}}{r_{1}}\right)
$$

e quindi

$$
i_{1}^{\prime}=\frac{\mathrm{E}}{r_{1}}-\frac{r}{r+m^{2} r_{1}}\left(m \mathrm{I}_{\tau}-\frac{\mathrm{E}}{r_{1}}\right) e^{-\alpha t}
$$

Si riconosce da ciò che per il fatto che il secondario è chiuso sul ricevitore la corrente primaria gia dal primo istante raggiunge un certo valore, continuando poi a crescere esponenzialmente fino al valore di regime $\frac{\mathbf{E}}{r_{1}}$. Ma il valore iniziale rapidamente raggiunto è eguale a

$$
\frac{\mathrm{E}}{r_{1}}-\frac{r}{r+m^{2} r_{1}}\left(m \mathrm{I}_{\tau}+\frac{\mathbf{E}}{r_{1}}\right)
$$

qualora la chiusura provochi una magnetizzazione opposta alla precedente, mentre ̀̀ eguale a

$$
\frac{\mathrm{E}}{r_{1}}-\frac{r}{r+m^{2} r_{1}}\left(m I_{\tau}-\frac{\mathrm{E}}{r_{1}}\right)
$$

quando la nuova magnetizzazione è nello stesso senso della primitiva. In quest' ultimo 'caso perciò la corrente primaria sarà più intensa fin dalla chiusura e conserverà valori più alti fino 
alla rottura, ciò che come vedremo attenua il trasferimento di energia dal primario al secondario, mentre accresce le perdite ohmiche nel primario.

Ma di maggior interesse è lo studio della corrente secondaria, governata come si è visto dalla equazione

$$
r i_{2}+\mathrm{L} \frac{r+m^{2} r_{1}}{r_{1}} \frac{d i_{2}}{d t}=-a
$$

e quindi dalla relazione

$$
i_{i}=\mathrm{C}+\mathrm{D} e^{-a l}
$$

con

$$
\alpha=\frac{r r_{1}}{\mathbf{L}\left(r+m^{2} r_{1}\right)}
$$

Per considerare il caso ordinario in cui la chiusura trovi nel secondario una corrente $I_{\tau}$ derivante dalla rottura anteriore, e tener conto che la presenza del tubo con la sua caratteristica equivale a quella di una forza contro elettromotrice $a$, si osservi che dopo un tempo lunghissimo si dovrebbe avere nel caso di una forza contro elettromotrice di senso costante

e perciò dev'essere

$$
i_{2}=-\frac{a}{r}
$$

$$
\mathrm{C}=-\frac{a}{r}
$$

Questo valore di $\mathrm{C}$ è esatto anche nel caso del tubo, considerando la $i_{2}$ fino al momento in cui essa.si annulla.

Se poi si indica con $I_{2}$ il valore che assume la corrente secondaria dopo qualche istante dalla ehiusura, si riconosce che dev'essere

$$
i_{2}=-\frac{a}{r}+\left(\mathrm{I}_{3}+\frac{a}{r}\right) e^{-l}
$$

poichè deve essere per $t=o$ (chiusura)

e per $t$ grandissimo

$$
i_{2}=\mathrm{I}_{2}
$$

$$
i_{2}=-\frac{a}{r}
$$


Per determinare $I_{2}$ si osservi che, se il processo di chiusura si svolgesse in pieno, la quantità di elettricità passata per gli effetti induttivi dovrebbe essere pari alla variazione del flusso divisa per la resistenza.

Ma la quantità di elettricità dovuta al processo induttivo risulta dal termine esponenziale di $i_{\mathrm{z}}$ integrato fra $o$ e $\infty$, ed è perciò eguale a

$$
\frac{\mathrm{I}_{2}+\frac{a}{r}}{\alpha}=\left(\mathrm{I}_{2}+\frac{a}{r}\right) \frac{\mathrm{L}\left(r+m^{2} r_{1}\right)}{r r_{1}} .
$$

Il flusso varia da $\mathrm{L}_{2} \mathrm{I}_{\tau} \mathrm{a}-\mathrm{M} \frac{\mathrm{E}}{r_{1}}-\mathrm{L}_{2} \frac{a}{r}$, poichè, sostituendo al tubo una pila di f.e.m. $-a$, la corrente finale nel secondario sarebbe $-\frac{a}{r}$.

Si può quindi scrivere

$$
\left(\mathrm{I}_{3}+\frac{a}{r}\right) \frac{\mathrm{L}\left(r+m^{2} r_{1}\right)}{r r_{1}}=\frac{\mathrm{L}_{8} \mathrm{I}_{\tau}+\mathrm{M} \frac{\mathrm{E}}{r_{1}}+\mathrm{L}_{2} \frac{a}{r}}{r}
$$

da eni, ponendo

$$
\mu=\frac{r}{r_{1}}+m^{2} \quad, \quad \mathrm{~V}_{\tau}=a+r \mathrm{I}_{\tau}
$$

e ricordando che si ha

$$
\mathrm{M}=m \mathrm{~L} \quad, \quad \mathrm{~L}_{2}=m^{2} \mathrm{~L},
$$

si ottiene

o anche

$$
r \mathrm{I}_{2}=-a+\frac{m^{2}}{\mu} \mathrm{V}_{\tau}+m \frac{r}{\mu} \frac{\mathrm{E}}{r_{1}}
$$

(3) $\mathrm{I}_{2}=-\frac{a}{r}+\frac{m^{2}}{\mu r} \mathrm{~V}_{\tau}+\frac{m}{\mu} \frac{\mathrm{E}}{r_{1}}=-\frac{a}{r}+\frac{m^{2}}{\mu r}\left(a+r \mathrm{I}_{\tau}\right)+\frac{m}{\mu} \frac{\mathrm{E}}{r_{1}}$.

Cosicchè l'effetto della chiusura sarà di portare rapidissimamente la corrente $I_{\tau}$ già esistente nel secondario al valore $I_{2}$. Che se la chiusura fosse avvenuta in modo da magne- 
tizzare il nucleo nel medesimo verso, allora si sarebbe ottenuta una corrente iniziale $I^{\prime}$, data da

$$
\mathrm{I}_{\mathrm{s}}^{\prime}=-\frac{a}{r}+\frac{m^{2}}{r \mu} \mathrm{V}_{\tau}-\frac{m}{\mu} \frac{\mathrm{E}}{r_{1}}=-\frac{a}{r}+\frac{m^{2}}{\mu r}\left(a+r \mathrm{I}_{\tau}\right)-\frac{m}{\mu} \frac{\mathrm{E}}{r_{\mathrm{v}}} .
$$

Dai valori iniziali $I_{2}$ o $\mathrm{I}^{1}$, la corrente decresce fino a zero secondo la legge rappresentata dalla (2) che corrisponde a una esponenziale di ampiezza $\mathrm{I}_{2}+\frac{a}{r}$ riferita a un'asse delle ascisse spostato in alto di $\frac{a}{r}$, e perciò di andamento ripido, quasi rettilineo.

Le migliori condizioni sperimentali, per quanto riguarda il ricavo di correnti secondarie intense, si otterranno quando è maggiore il valore di $I_{2}$. E si riconosce senz'altro che a parità di condizioni è sempre

$$
\mathrm{I}_{2}>\mathrm{I}_{2}^{\prime}
$$

e che perciò la chiusura con magnetizzazione invertita sarà sempre preferibile a quella con magnetizzazione diretta rispetto alla precedente.

Ma anche la chiusura a magnetizzazione invertita potrà non dare i suoi effetti più efficaci, qualora non siano convenientemente scelti gli elementi da cui dipende il valore di $I_{2}$.

Essi sono, per un dato tubo, $\mathbf{I}_{\tau}, m$ ed $\mathbf{E}$.

Il valore di $I_{\tau}$, corrente residua secondaria al momento della chiusura, dipende dalla durata della pausa fra l'apertura e la chiusura, e dalla durezza del tubo, nel senso che se questo è molto duro la corrente secondaria di rottura, che si svolge anch'essa quasi secondo una retta d'inclinazione variabile, si sard ridotta di molto nel tempo della pausa. Se il tubo è molle e la pausa è breve, la chiusura sopravviene mentre la corrente secondaria, e quindi $I_{\tau}$, non è molto diminuita dal valore iniziale. La formola ei dice intanto che $I_{2}$ è diverso da $I_{\tau}$, ma sempre maggiere dal valore che avrebbe avuto se $I_{\tau}$ fosse nulla, cioè se la chiusura seguisse dopo una pansa molto lunga. 
La discussione della formola (3) si facilita se, invece di considerare l'intensità massima $I_{2}$ che si stabilisce poco dopo la chiusura, si prende in esame la tensione $V_{2}$ esistente ai poli del tubo, tensione che si può ritenere eguale a $r \mathrm{I}_{2}+a$, trascurando la perdita ohmica $r_{2} \mathrm{I}_{2}$ nel secondario.

Si ottiene dalla (3)

$$
\begin{aligned}
\mathrm{V}_{3} & =\frac{m}{\mu}\left(m \mathrm{~V}_{\tau}+r \frac{\mathrm{E}}{r_{1}}\right) \\
& =\frac{m^{2}}{m^{2}+\frac{r}{r_{1}}} \mathrm{~V}_{\tau}+\frac{r / r_{1}}{m^{2}+\frac{r}{r_{1}}} m \mathrm{E} .
\end{aligned}
$$

E questa formola ci dice che dopo la chiusura la tensione ai poli del tubo risulta da due frazioni complementari della tensione $V_{\tau}$ preesistente prima della chiusura e delia tensione $m \mathrm{E}$ che si desterebbe, nel secondario aperto per virtù della tensione $\mathbf{E}$ applicata br̀uscamente al primario.

Se il primario venissd chiuso in corto circuito, anzichè sulla tensione $\mathrm{E}, \mathrm{V}_{2}$ si ridurrebbe alla prima parte; se la chiusura sulla tensione $\mathrm{E}$ tardasse di tanto da potersi considerare esauIrta la precedente corrente di rottura, e quindi nullo $V_{\tau}$, sussisterebbe solo la seconda. Si vede da ciò che la chiusura con senso opposto di magnetizzazione mentre ancora soppravvive la precedente corrente di rottura esalta la tensione rispetto a quella propria della chiusura, ma può farla discendere al di sotto del valore $V_{\tau}$ esistente quando la chiusura avviene. Invero se si ha

risulta

$$
\begin{aligned}
& m \mathrm{E}<\mathrm{V}_{\tau} \\
& \mathrm{V}_{\mathrm{a}}<\mathrm{V}_{\tau} .
\end{aligned}
$$

In altri termini una chiusura intempestiva può nuocere in quanto deprime di colpo la tensione e quindi la corrente preesisterte; ciò darà effetti più gravi se $m$, coefficiente di moltiplicazione del rocchetto, è piccolo come nei rocchetti esistenti. Che se si dà a $\mathrm{E}$ ed $m$ un valore elevato, sarà possibile ottenere, anche con una chiusura rapidamente stabilita 
dopo la rottura, che la tensione e quindi la corrente preesistenti vengano esaltate anzichè depresse dalla chiusura.

Da alcuni diagrammi della corrente secondaria che saranno presto pubblicati dal dott. Trabacchi apparira nettamente confermata dall'esperienza la previsione fatta sulla influenza della durata della pausa, della tensione primaria e del coefficiente di moltiplicazione, ottenendosi così la giustificazione degli ottimi risultati pratici raggiunti con l'apparecchio radiologioo costruito.

Ma la semplice considerazione della intensita e della tensione secondaria va integrata, per un esame completo della questione, con lo studio della energia complessiva ricavabile al secondario per effetto di una rottura seguita da una chiusura, poichè se questa avviene intempestivamente, quando sussiste ancora una notevole energia magnetica nel nucleo tuttora magnetizzato dalla corrente secondaria non esaurita, sembra che, sostituendosi al libero svolgimento nel tubo della energia di rottura la nuova chiusura, debba derivarne una perdita dell'energia totale trasferita al secondario.

Per valutare l'energia complessiva $W_{z}$ ricavata al secondario come effetto di una rottura e di una chiusura operata quando l'intensità di rottura ha ancora il valore $I_{\tau}$, osserviamo che la rottura esplicantesi in pieno libererebbe una energia totale $\frac{\mathrm{LI}_{1}{ }^{2}}{2}$, dove $\mathrm{I}_{1}$ è la corrente primaria all' istante della rottura; ma di qnesta una parte $m^{2} \frac{L_{1} I_{\tau}{ }^{2}}{2}$ è ancora concentrata nel nucleo magnetico al momento della chiusura; cosicchè indicando con $W_{c}$ la totale energia svolta nella fase di chiusura, si avrì

$$
\mathrm{W}_{2}=\frac{\mathrm{LI}_{1}^{2}}{2}-m^{2} \frac{\mathrm{L} \mathrm{I}_{\tau}^{2}}{2}+\mathrm{W}_{c}
$$

Per valutare $W_{c}$ si consideri la (1) che può essere scritta

$$
\mathrm{L} \frac{r+m^{2} r_{1}}{r_{1}} \frac{d i_{8}}{d t}=-\left(a+r i_{2}\right)=-v
$$


dove $v$ denota la tensione agli estremi del ricevitore, qualora si trascuri la resistenza ințerna $r$, del secondario.

Moltiplicando per $i_{2}$ e integrando fra $o$ e $t$ si ottiene

$$
\frac{1}{2} \mathrm{I} \cdot \frac{r+m^{2} r_{1}}{r_{1}}\left(\mathrm{I}_{2}^{2}-\mathrm{I}_{1}^{2}\right)=\int_{0}^{t} v i_{2} d t
$$

dove $I_{q}$ denota la corrente secondaria iniziale e $I_{l}$ la corrente secondaria al tempo $t$.

Se si sceglie $t$ in modo che sia divenuto $\mathrm{I}_{t}=0 ;$ cioè che sia cessata la corrente secondaria, il secondo membro diviene la potenza $W_{c}$ svolta nel secondario; si ha perciò

$$
\mathrm{W}_{c}=\frac{1}{2} \mathrm{~L} \frac{r+m^{2} r_{1}}{r_{1}} \mathrm{I}_{2}^{2}=\frac{1}{2} \mathrm{~L}_{\mu} \mathrm{I}_{2}^{2} \text {; }
$$

e poichè abbiamo nella (3) il valore di $I_{2}$ sarà facile dedurre $W_{c}$.

Sostituendone il valore in (4) si ottiene per la energia totale $\mathrm{W}_{2}$, dopo alcuni facili calcoli,

$$
\begin{aligned}
\mathrm{W}_{2} & =\frac{\mathrm{L}}{2}\left[\mathrm{I}_{1}{ }^{3}+\frac{r_{1}}{r+m^{3} r_{1}}\left(\frac{m \mathrm{E}-a}{r_{1}}\right)^{2}+\right. \\
& \left.+\frac{m^{2}}{r+m^{2} r_{1}} \mathrm{I}_{\tau}\left\{2(m \mathrm{E}-a)-2 \mathrm{I}_{\tau}\right\}\right] .
\end{aligned}
$$

Come si riconosce, $W_{2}$ risulta di tre parti: la prima, rappresentata dal $1 .^{\circ}$ termine, corrisponde alla energia che si ricaverebbe dalla sola rottura se questa si svolgesse fino allesaurimento della 'corrente secondaria: la seconda, data dal $2 .^{0}$ termine, misura l'energia corrispondente a una chiusura isolata; l'insieme dei primi due termini darebbe l'energia dovuta a una rottura seguita da lunghissima pausa e dalla seguente chiusura $\left(I_{-}=0\right)$; finalmente il terzo termine fornisce l'eccesso di energia derivante dal fatto che la chiusura con magnetizzazione opposta avviene mentre sopravvive ancora, col valore $I_{\tau}$, la corrente di rottura.

Ma questo termine aggiuntivo non è sempre favorevole. Infatti se fosse

$$
2(m \mathrm{E}-a)<r \mathbf{I}_{\tau}
$$


il terzo termine diverrebbe negativo, e l'energia ottenuta sarebbe minore di quella spettante a una rottura e a una chiusura molto distanti nel tempo. Questa azione ostacolatrice della intempestiva chiusura, per un dato $I_{\tau}$, si produrrà tanto più facilmente quanto più sono piccoli $m$ ed $\mathbf{E}$.

Ma anche quando il terzo termine è positivo, la sua entità può esser diversa, a seconda del tempo di pausa, da cui dipende $\mathbf{I}_{\tau}$, e dei valori di $m$ e di $\mathbf{E}$. Il valore più favorevole si ottiene risolvendo un semplice problema di massimo per la espressione

$$
\mathbf{I}_{\tau}\left[2(m \mathbf{E}-a)-r \mathbf{I}_{\tau}\right]
$$

nei riguardi di $I_{\tau}$, qualora si voglia determinare la durata più vantaggiosa di pausa, o il valore più conveniente di $I_{\tau}$. Si ottiene così

$$
m \mathrm{E}=a+r \mathrm{I}_{\tau}=\mathrm{V}_{\tau}
$$

occorre ciò̀ che la tensione di chiusura a circuito aperto, $m \mathrm{E}$, sia eguale alla tensione totale $V_{\tau}$ esistente nel secondario. Più è breve la pausa, e più è elevata la tensione secondaria superstite $V_{\tau}$, al momento della rottura, più dovrà esser grande il coefficiente di moltiplicazione $m$ del rocchetto, se si vuole che l'energia totale superi notevolmente quella che spetterebbe allo insieme di una rottura e di una chiusura che non si sovrappongano insieme. Che se il coefficiente di moltiplicazione $m$ e la tensione E non sono abbastanza elevati, non solo si perderanno i vantaggi della compenetrazione dei processi di rottura e di chiusura, ma si potrà anche ottenere meno di quello che produrrebbe una rottura seguita senza compenetrazione da una chiusura.

Il non aver tenuto conto di queste particolari condizioni che posson rendere nocivo anzichè utile il sistema di far funzionare il rocchetto a magnetizzazioni successivamente invertite spiega l'insuccesso di altri sperimentatori che hanno tentato di usare il medesimo artificio. Le ricerche che saranno pubblicate fra breve dal dott. Trabacchi, e nelle quali sono stati 
rilevati i diagrammi della corrente secondaria, e l'azione radiografica delle singole scariche nelle fasi susseguentisi di apertura e di chiusura metteranno ancora più efficacemente in rilievo i punti già segnati dalla teoria, e le conclusioni che da questa si sono dedotte nel presente lavoro. 